\title{
Specification of the near-Earth space environment with SHIELDS
}

V. K. Jordanova ${ }^{\mathrm{a}, *}$, G. L. Delzanno ${ }^{\mathrm{a}}$, M. G. Henderson ${ }^{\mathrm{a}}$, H. C. Godinez ${ }^{\mathrm{a}}$, C. A. Jeffery ${ }^{\mathrm{a}}$, E. C. Lawrence ${ }^{\mathrm{a}}$, S. K. Morley ${ }^{\mathrm{a}}$, J. D. Moulton ${ }^{\mathrm{a}}$, L. J. Vernon ${ }^{\mathrm{a}}$, J. R. Woodroffe ${ }^{\mathrm{a}}$, T. V. Brito ${ }^{\mathrm{a}}$, M. A. Engel ${ }^{\mathrm{a}}$, C. S. Meierbachtol ${ }^{\mathrm{a}}$, D. Svyatsky ${ }^{\mathrm{a}}$, Y. Yu ${ }^{\mathrm{a}, 1}$, G. Tóth ${ }^{\mathrm{b}}$, D. T. Welling ${ }^{\mathrm{b}}$, Y. Chen ${ }^{\mathrm{b}}$, J. Haiducek ${ }^{\mathrm{b}}$, S. Markidis ${ }^{\mathrm{c}}$, J. M. Albert ${ }^{\mathrm{d}}$, J. Birn ${ }^{\mathrm{a}, \mathrm{e}}$, M. H. Denton ${ }^{\mathrm{e}, \mathrm{f}}$, R. B. Horne ${ }^{\mathrm{g}}$

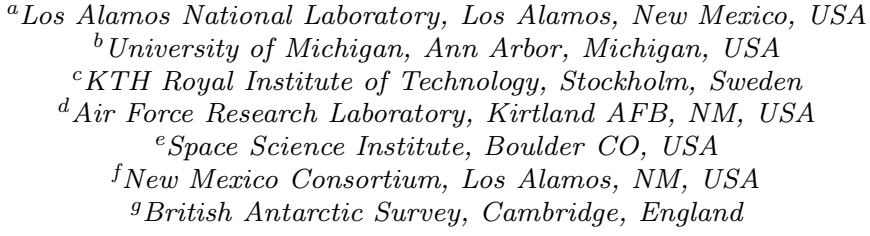

\begin{abstract}
Predicting variations in the near-Earth space environment that can lead to spacecraft damage and failure is one example of "space weather" and a big space physics challenge. A project recently funded through the Los Alamos National Laboratory (LANL) Directed Research and Development (LDRD) program aims at developing a new capability to understand, model, and predict Space Hazards Induced near Earth by Large Dynamic Storms, the SHIELDS framework. The project goals are to understand the dynamics of the surface charging environment $(\mathrm{SCE})$, the hot $(\mathrm{keV})$ electrons representing the source and seed populations for the radiation belts, on both macro- and micro-scale. Important physics questions related to particle injection and acceleration associated with magnetospheric storms and substorms, as well as plasma waves, are investigated. These challenging problems are addressed using a team of world-class experts in the fields of space science and computational plasma physics, and state-of-the-art models and computational facilities. A full two-
\end{abstract}

\footnotetext{
* Corresponding author

Email addresses: vania@lanl.gov (V. K. Jordanova)

${ }^{1}$ Now at Beihang University, Beijing, China.
} 
way coupling of physics-based models across multiple scales, including a global MHD (BATS-R-US) embedding a particle-in-cell (iPIC3D) and an inner magnetosphere (RAM-SCB) codes, is achieved. New data assimilation techniques employing in situ satellite data are developed; these provide an order of magnitude improvement in the accuracy in the simulation of the SCE. SHIELDS also includes a post-processing tool designed to calculate the surface charging for specific spacecraft geometry using the Curvilinear Particle-In-Cell (CPIC) code that can be used for reanalysis of satellite failures or for satellite design.

Key words: geomagnetic storms and substorms, multiscale physics, wave-particle interactions, space hazards, numerical modeling

\section{Introduction}

Our society is increasingly dependent on satellite-based technologies (broadcast TV/Radio, cell phones, GPS, internet, commercial/military/national security assets) susceptible to harmful conditions in space, i.e. "space weather" [1].

5 The introduction of all-electric propulsion satellites in the quest for low-cost access to space raises new questions regarding the threat of adverse space weather [2]. In addition, ground based technologies such as electric power grids, high voltage transmission lines, and pipelines are affected by space weather. Through disruption of power grids, communications, and satellite operations, severe geomagnetic storms may cause damage costing $\$ 1$ to $\$ 2$ trillion during the first year alone and taking years for recovery [3]. Therefore, forecast of severe space weather events would be very valuable to allow the communication/operation centers to prepare in advance and to take protective actions [4, 5].

The near-Earth inner magnetosphere, where most of the nation's civilian and 15 military space assets operate, is an extremely hazardous region of the space environment which poses major risks to our space infrastructure. As one example, Galaxy 15, a $\$ 250$ million telecommunication satellite in geosynchronous orbit (GEO), failed to operate in 2010 due to a space storm, and its recovery operation cost about $\$ 3.5$ million $[6]$. Failure of satellite subsystems or even total failure 
of a spacecraft can arise for a variety of reasons. Some of these are related to the space environment including space weather events like single-event-upsets and deep dielectric charging caused by high energy particles, or surface charging caused by low to medium energy particles. Other space hazards are collisions with natural or man-made space debris, or intentional hostile acts. The ability to reliably distinguish between these modes of failure is critically important in anomaly resolution and forensics.

Reliable modeling of the space environment and predicting space weather hazards is, however, a longstanding challenge due to the complex multiscale nature of the magnetosphere. The Earth's magnetosphere covers a vast region of space dominated by the Earth's magnetic field. It is a highly dynamic system that responds nonlinearly to driving by the time-varying solar wind, being coupled through a variety of processes over a broad range of physical scales. Conditions in space change quickly from quiet to harmful and geomagnetic disturbances may last for days. Storms strengthen the ring current which consists of magnetically trapped charged particles $(\sim 1-100 \mathrm{keV})$; these particles drift around Earth between $\sim 2$ to 5 Earth radii $\left(R_{E}\right)$. Substorms, wherein the nightside magnetosphere reconfigures on a timescale of few minutes occur several times per day, releasing fast plasma flows and injecting hot ( $\sim 10$ 's keV) electrons into the near-Earth region. The anisotropic particle injections lead to

40 the generation of plasma waves which transfer energy from the fields back to the particles. Charged particles can be pumped to high energies that damage spacecraft, or can be precipitated into the Earth's atmosphere. This extremely complex feedback mechanism balances the source and loss processes and regulates the distribution of particle populations in the near-Earth space.

45

An end-to-end model of the magnetosphere which addresses these fundamental space physics problems and aims at specifying Space Hazards Induced near Earth by Large Dynamic Storms, the SHIELDS framework, is being developed at Los Alamos National Laboratory (LANL). SHIELDS is driven by the dynamic solar wind and simulates one of the most harmful space weather hazards 50 - the spacecraft surface charging environment (SCE) [7]. A thorough under- 
standing of the SCE is needed to strengthen spacecraft design and minimize the susceptibility of satellites to space weather. As satellites orbit around the planet, they are bombarded with charged particles, and surface charging results from the collection of charged particles by the spacecraft. Surface charging can lead to potential differences across spacecraft components and cause discharges that can damage electronics. The primary SCE source is the low-energy (10's of $\mathrm{keV}$ ) hot electrons injected from the magnetotail into the inner magnetosphere during storms and substorms. SHIELDS simulates the dynamics of the hot particles (the seed population for the radiation belts) including important physics of magnetic reconnection, storm/substorm injection, and wave-particle interactions. The framework fully integrates several physics-based models across multiple scales, from macro-scale (global MHD, BATS-R-US), and meso-scale (inner magnetosphere, RAM-SCB), to micro-scale (particle-in-cell, iPIC3D). New data assimilation techniques employing data from LANL instruments on the

${ }_{65}$ Van Allen Probes are applied for the first time to the inner magnetosphere model, showing very promising results. For the past 50 years, LANL has built and designed space-based sensors, many of which have been used to study space weather, as understanding and predicting such phenomena are important to national security. SHIELDS makes use of these data to provide an accurate global 70 specification of the SCE for the most heavily satellite-populated region of the Earth's magnetosphere.

\section{Description of the SHIELDS Framework and its Applications}

SHIELDS uses numerical models as powerful tools to specify space weather globally and to place sparsely available space measurements into global context.

75 Given the complex multiscale physics in the magnetosphere, analysis and understanding SCE dynamics requires models that are targeted at key regions/physics regimes. However, the coupling of these models across multiple spatial and temporal scales remains an extreme challenge. SHIELDS leverages from the University of Michigan Space Weather Modeling Framework (SWMF, [8]), that 
integrates interoperating models of physics domains into a high-performance coupled model. Major SHIELDS developments (Figure 1) are the addition to the SWMF of new kinetic models - iPIC3D [9] and RAM-SCB [10] and data assimilation tools [11]. Such additions provide crucial coupling to microphysics responsible for the SCE, thereby obtaining an improved specification of magnetic reconnection, storm/substorm injections, and plasma wave dynamics. The models are coupled together by the framework code including a control module that determines the overall time-stepping and communication between the models. The control module also determines when the coupling among models should occur, the order in which it happens, and it takes care of grid interpolation and synchronization of the model runs to allow for a physically meaningful coupling. Figure 1 shows a flow chart of how the various macro- and micro-scale models used in SHIELDS are inter-connected and how the information is exchanged among them. Further details on SHIELDS advancements are presented below.

\subsection{Reconnection physics}

In the SHIELDS project, the global magnetosphere is modeled with the Block Adaptive Tree Solar wind Roe-type Upwind Scheme (BATS-R-US) [12, 13] MHD code developed at the University of Michigan. Magnetohydrodynamic (MHD) codes provide a realistic global context for the more process/region specific models. They can model the three-dimensional (3D) magnetosphere with reasonably fine grid resolution faster than real time on a small cluster (i.e., to simulate 1 day of magnetospheric activity requires less than a day in computational time). BATS-R-US is driven by solar wind data applied as upstream boundary conditions (at $\sim 30 R_{E}$ ); the other boundaries (at $\sim 100$ to $300 R_{E}$ ) let the plasma flow out of the domain. The inner boundary conditions are provided by the Ridley Ionosphere Model (RIM) [14] driven by field aligned currents (FAC) from BATS-R-US. A non-trivial challenge for global MHD models due to the missing microphysics, however, is that typically they cannot reproduce the fast reconnection rates observed in kinetic simulations. On the other hand, 


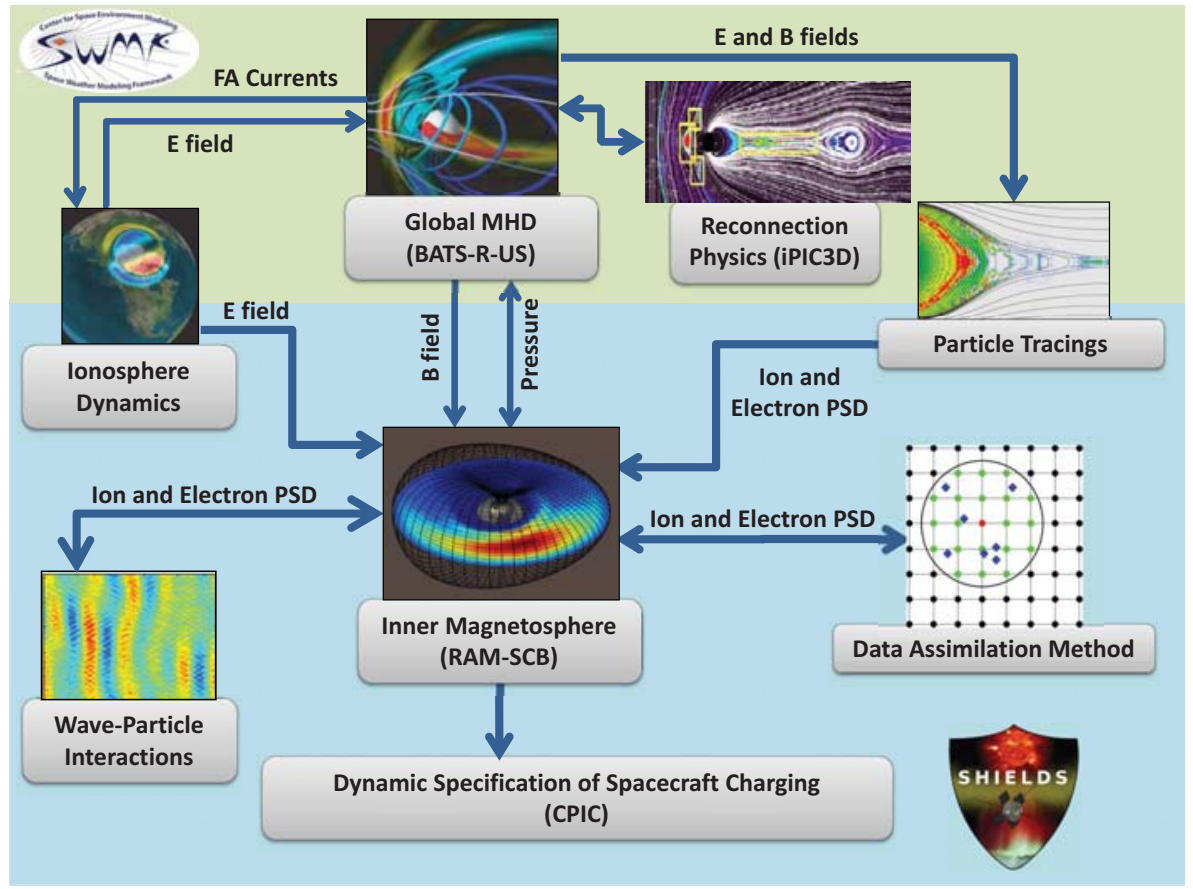

Figure 1: Schematic flow chart of the comprehensive SHIELDS framework showing how this software connects macro- and micro-scale models and combines the results with dataassimilation tools to capture the severity of the spacecraft charging environment. 
particle-in-cell (PIC) codes have been used with great success in studies of kinetic phenomena like magnetic reconnection. PIC codes solve the full set of Maxwell's equations for the electromagnetic fields, coupled with the equations of motion for electrons and ions. However, they are usually restricted to local simulations due to their high computational cost.

To address this challenge, the BATS-R-US code [13] was two-way coupled with a regional PIC code [9], thus obtaining an exceptional capability, the MHD with Embedded PIC (MHD-EPIC) algorithm [15]. The PIC code covers the regions where kinetic effects are important while the rest of the domain is handled by the fluid code. The implicit particle-in-cell code iPIC3D [9] was integrated into the SWMF as the first model representing the new Particle-in-Cell (PC) component. A general parallel coupler was implemented since the existing SWMF couplers were not suitable for passing the large amount of data between the two massively parallel models. The new coupler keeps the grid description and interpolation methods private for the models. This eliminates the need to describe the grid in an abstract manner and to pass this information to the SWMF or the coupled model. The coupling is very efficient and takes less than $2 \%$ of the execution time in all simulations. The coupling between BATS-RUS and iPIC3D works in 3D, and the two grids do not need to be aligned. In addition, multiple PIC regions may be used. This new efficient and flexible coupler was used to model Ganymede's magnetosphere in 3D with 4 PIC regions. The results gave excellent agreement with Galileo measurements, much better than with Hall MHD, showing that the MHD-EPIC algorithm provides a better description of reconnection than the fluid models [16].

Most recently we have applied MHD-EPIC to the Earth [17], which is the main target for the SHIELDS project. This is a very challenging problem, because the ion inertial length, the spatial scale magnetic reconnection originates at, is very small compared to the system size. In an initial study, we have put the PIC box around the dayside reconnection site, as it is smaller than the tail reconnection. Even this is very expensive unless we artificially increase the kinetic scales by changing the ion mass per charge of particles. To check the 


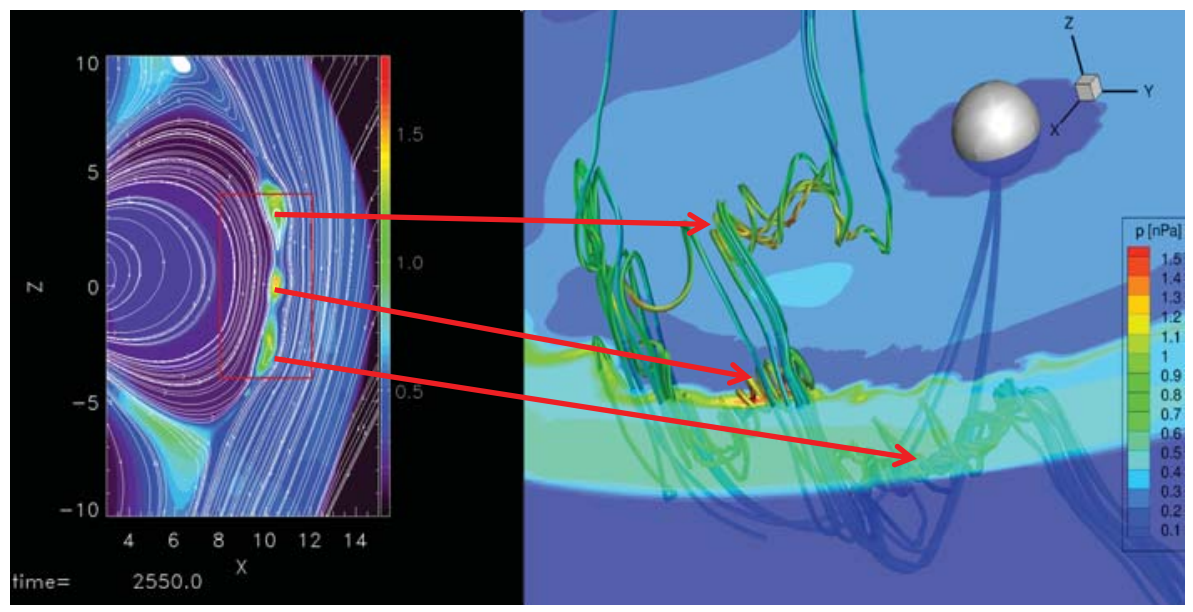

Figure 2: The FTEs from MHD-EPIC simulation of the Earth's magnetosphere at $\mathrm{t}=2550 \mathrm{~s}$. The left panel shows the pressure (nPa) and the projected magnetic field lines at the meridional plane. The red box represents the region covered by the PIC code. The right panel is the 3D view at the same time. The plasma pressure at the $\mathrm{z}=0$ plane, and the FTEs colored by pressure are shown.

effect of ion mass per charge on the global solution, we performed a series of $2 \mathrm{D}$ MHD-EPIC simulations for an Earth-like system [18]. We found that the results are insensitive, suggesting that we can increase the ion mass per charge and still obtain physically correct solutions on the resolved scales. Using this idea, we performed 3D MHD-EPIC simulations [17] with an ion mass per charge set to 16. The simulation worked very robustly and efficiently. We thus simulated an hour of real time with constant solar wind driving (with negative $B_{z}$ ) and found several flux ropes forming at the dayside reconnection site covered by the PIC region. Figure 2 shows a three-dimensional example of this MHDEPIC simulation. The dayside magnetopause is covered by a PIC box, which is represented by the red box in the left panel of Figure 2 Magnetic reconnection happens inside the PIC box and generates flux transfer events (FTEs). The right panel shows a 3D view of three FTEs. Several global and small scale phenomena that are properly reproduced by the simulation are described in [17], including virtual satellite observation of FTEs, signatures of the lower hybrid 
drift instability (LHDI), and the crescent shape velocity distribution functions observed by the MMS spacecraft.

Thanks to the MHD-EPIC algorithm and the increased ion mass per charge we could perform this global simulation with a relatively small and coarse PIC grid (about $1 / 30 R_{E}$ ) while still properly capturing the kinetic reconnection process. This simulation required only 2000 core hours per minute, about 65,000 times faster than what it would take to run with the proton mass per charge ratio and another factor of 10,000 faster than what it would take to run with a global PIC model. Further work using the MHD-EPIC model to study the substorm process where the PIC region covers the Earth's tail reconnection is ongoing.

\subsection{Storm/substorm dynamics}

The injection of hot $(\mathrm{keV})$ particles into the inner magnetosphere is enhanced during geomagnetic storms and substorms. Geomagnetic storms, one of the most important space weather events, are usually triggered by plasma eruptions on the Sun that travel through interplanetary space, reaching Earth in 1 to 4 days and lasting several days [19]. By contrast, substorms are characterized by a rapid (on time scales of minutes) reconfiguration of the near-Earth magnetic field, releasing a huge amount of energy as a violent, Earthward-directed plasma flow [20]. Substorms typically recover within a few hours and occur, on average, every 5 hours [21]. An important step for the successful modeling of geomagnetic storm dynamics was the two-way coupling of global MHD models with inner magnetosphere "drift codes" [22, 23]. MHD simulations have great potential for forecasting substorm effects, since the magnetic field perturbations due to substorm-associated currents can be calculated directly from an MHD result. In addition, global MHD is already being used operationally at NOAA/SWPC to forecast magnetic field perturbations at the Earth's surface. The ability of MHD to produce realistic substorms has been tested for case studies [24, 25]. However, no MHD model has yet been validated with regard to its ability to 
as would likely be the case for operational applications.

We are evaluating the capabilities of MHD to reproduce the onset times of observed substorms. We simulate the entire month of January 2005 using the SWMF [8] in a configuration very similar to that described in [26]. The only inputs to the model are observed solar wind parameters obtained from NASA/GSFC's 1-minute OMNI dataset and the Advanced Composition Explorer (ACE) spacecraft [27], and NOAA F10.7 flux. We identify substorms in the model output and in observational data from the same time period. To ensure correctness in the event identification, we identify substorms using multiple datasets and identification techniques, and only events which are corroborated by multiple techniques are included in the final analysis. Using Biot-Savart integrals over the MHD domain the model delivers predictions of magnetic field at the Earth's surface, enabling us to calculate values of geomagnetic indices for comparison with observations. The datasets used for identification include the auroral lower (AL) index, midlatitude postive bay (MPB) index [28], and in situ dipolarization signatures. We use the Supermag algorithm to identify AL onsets in both the model and the observations, while in the case of the MPB index we use the procedure given in [28]. In the model output we also include plasmoid releases (identified by visual inspection of the model output). In the observations we additionally include IMAGE-FUV substorms (using the list provided by [29]), and dispersionless particle injections identified by inspection of data from the LANL SOPA instrument [30]. An example of one of the substorms is shown in Figure 3. The upper panel of Figure 3 shows a plasmoid release from the model output, with closed field lines (those that connect to the Earth at two points in these 2-D traces) drawn in white and open field lines (those that connect to the Earth at one end, or not at all) in black. The plasmoid is identified by a region of spiraling field lines in the tail region. The lower panel of Figure 3 shows observed $B_{z}$ at GOES 10 [31] along with model output for the satellite location. The magnetic field at GOES 10 shows a dipolarization whose onset coincides with the plasmoid release, which is a typical geosynchronous signature of a substorm [32]. Analysis of model output and observational data 

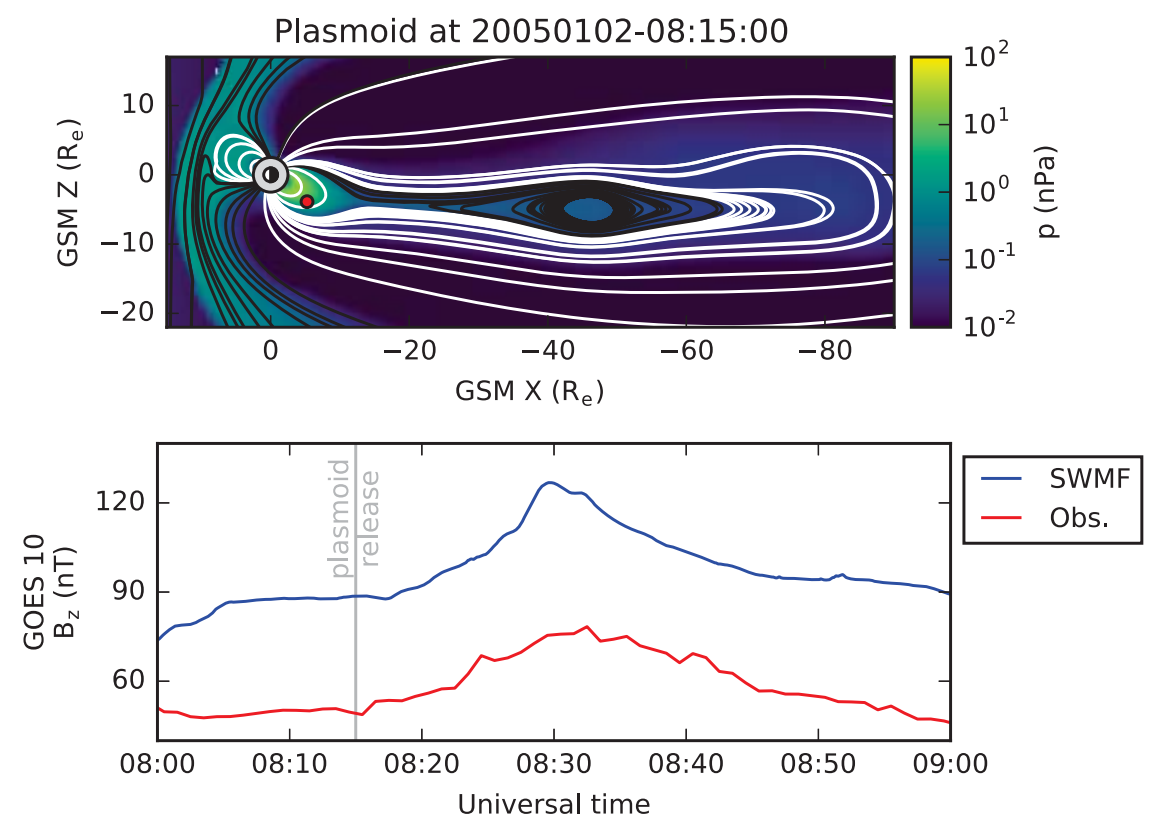

Figure 3: Substorm signatures for an event occurring around 0815 UT on January 2, 2005. The top panel shows a plasmoid release in the model output. The red dot in the plot denotes the location of GOES 10. Magnetic field at GOES 10 is plotted in the bottom panel, showing a dipolarization coincident with the plasmoid release.

was performed using the Spacepy software package [33].

We break the month into 30-minute bins, and categorize each bin as a "hit" if a substorm occurred in both model and observations within the bin, a "miss" 220 if a substorm occurred only in the observations, a "false positive" if a substorm occurred only in the model and a "true negative" if a substorm occurred in neither model nor observations. In total, we find 118 substorms in the model output, and 163 in the observations. Of these there were 29 hits, 134 misses, 89 false positives, plus 1236 true negatives. From this we calculate a Heidke 225 skill score [34] of 0.126. Positive values for the Heidke skill score (such as we have) indicate a predictive capability better than a random reference forecast, while a Heidke skill score of 1 would indicate a perfect forecast. Determining uncertainty bounds for the Heidke skill score is non-trivial [35] and there is no 
known analytical formula to do so. In future work, we plan to estimate the US coupled with RAM-SCB [40], until they reached a distance of $-15 R_{E}$ in the tail. The distribution function at the tailward boundary was determined by combining an analytical kappa distribution [41] with parameters determined 


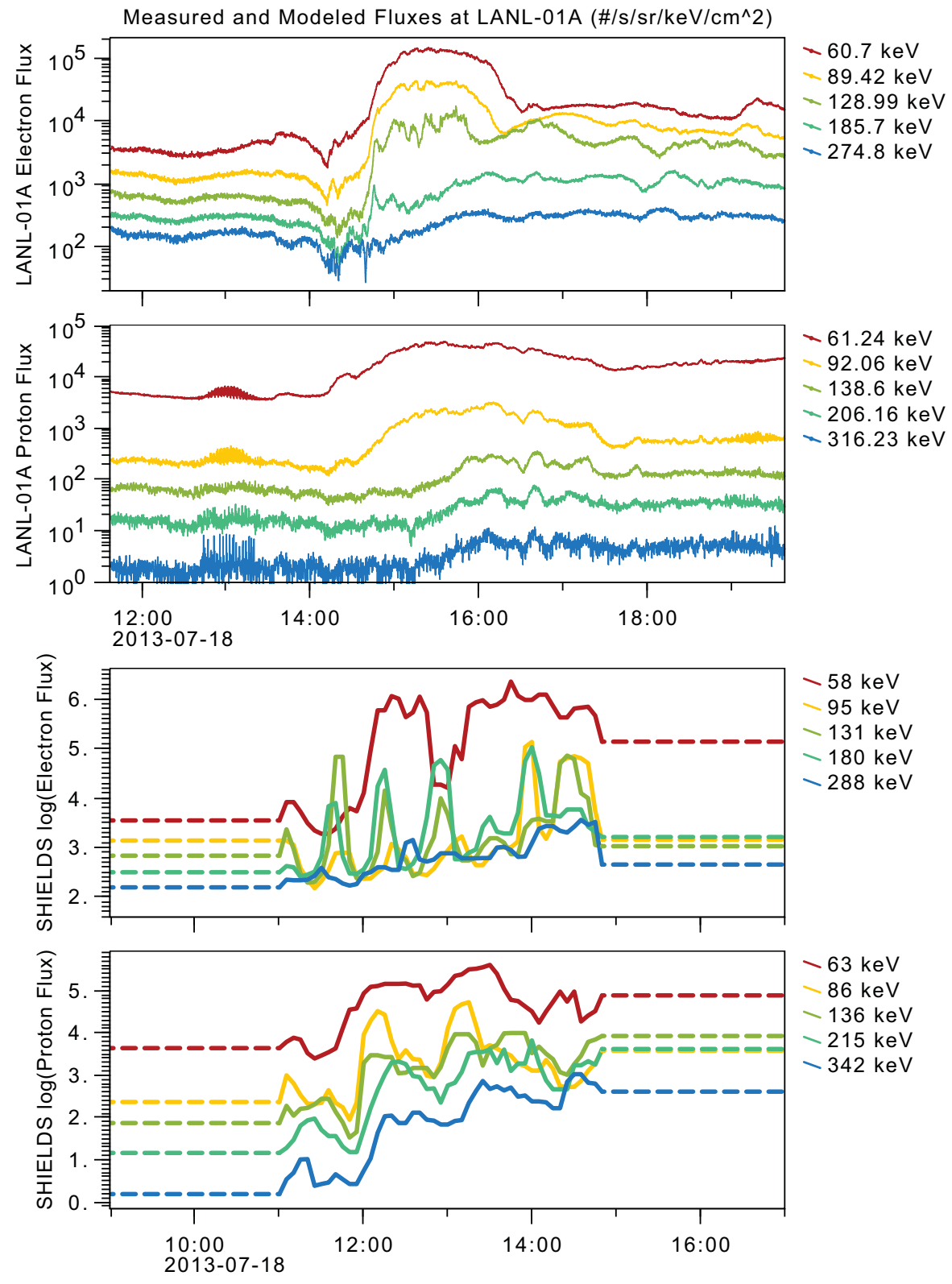

Figure 4: (top) Spin-averaged electron and ion fluxes measured at GEO with LANL MPA and SOPA instruments during a substorm event that occurred around 15 UT on July 18, 2013. (bottom) Simulations from the Particle Tracing Model (PTM) showing complex dispersive injection activity between $\sim 12$ and 15 UT. The simulated fluxes are normalized to the initial conditions indicated with dashed lines. 
from an empirical plasmasheet model 42]. The obtained distribution function was then mapped back to GEO using Liouville's theorem. This tracing was repeated every minute for several hours, resulting in the omnidirectional flux profile compared to in situ LANL spacecraft observations in Figure 4 . The time scale for both observations and simulation are the same, however they are shifted relative to one another since the simulation did not reproduce the injection time. Note that the timing difference is likely a result of the MHD simulation since it's a challenge for global MHD models to accurately describe when a substorm is going to occur based only on solar wind inputs. As explained in section 2.1, we are addressing this challenge by embedding a PIC module in BATS-R-US to allow reconnection physics to develop more realistically in MHD-EPIC; this will be explored in future work. Nevertheless, some of the dynamic features present in real particle injections, and specifically the rapid flux increases at lower energies, were qualitatively reproduced by the particle tracings. These flux enhancements are due to particle transport and acceleration caused by the magnetic field dipolarization and the associated electric field [43]. Future work will investigate the formation of injection boundaries and their depth of penetration toward the inner magnetosphere as a function of external driving conditions.

\subsection{Wave-particle interactions (WPI)}

Storms/substorms drive plasma waves that redistribute energy throughout the collisionless magnetospheric environment and couple low-energy plasma with high-energy particles. The addition of the effects of these waves requires models that can couple micro-scale wave-particle interactions with macro-scale ring current dynamics. Of prime interest to the SHIELDS investigations are whistlermode waves such as chorus and hiss that can efficiently scatter relativistic electrons in the inner magnetosphere [44]. Chorus waves are discrete emissions often containing rising and falling tones, most frequently observed during geomagnetically active times outside of the plasmasphere between $~ 23$ and 11 MLT from about 4 to $7 R_{E}$ [45]. On the other hand, hiss is a broadband whistler-mode 
emission mostly observed inside the plasmasphere and high-density plumes. Recent observations from the Van Allen Probes (previously known as the Radiation Belt Storm Probes, RBSP) show that the statistical hiss peak frequencies are typically between 100 and $300 \mathrm{~Hz}$ and that hiss wave power frequently extends below $100 \mathrm{~Hz}$, especially at larger radial distances on the dayside during enhanced levels of substorm activity [46].

We investigated the combined effects from time-dependent transport and scattering by whistler-mode chorus and hiss on the SCE using our magnetically self-consistent ring current-atmosphere interactions model (RAM-SCB) [10, 47]. This model solves the bounce-averaged kinetic equation for the hot (keV) ion and electron populations in the inner magnetosphere, taking into account all key source and loss processes. The magnetic field is calculated self-consistently with the anisotropic ring current plasma pressure [48] and used subsequently to propagate the particle distributions. At first, we investigated the electron precipitation to the atmosphere comparing two loss methods: (1) the "diffusion coefficient method" employing pitch angle diffusion coefficients determined from quasi-linear theory, with wave characteristics obtained from statistical observations, and (2) the "lifetime method" employing electron lifetimes independent of pitch angles inferred from the above diffusion coefficients. We found that both loss methods demonstrate similar temporal evolution of the trapped ring current electrons, however, the lifetime method hardly captures any precipitation at larger radial distances (i.e., $4<L<6.5$ ), while the diffusion coefficient method produces much better agreement with NOAA/POES and DMSP measurements (see Figure 8 of [49]).

In our next study, of the October 2012 "double-dip" storm [50], we included both pitch angle and energy scattering using quasi-linear theory and $L$ and MLT dependent event-specific chorus wave models inferred from NOAA/POES and Van Allen Probes observations (Figure 5 top). The spatial and temporal evolution of peak chorus wave amplitudes was in reasonable agreement with linear growth rate maxima calculated with RAM-SCB. For hiss waves inside the plasmasphere we used the empirical wave distributions statistically derived from 
the CRRES wave data [51, 52]. The dynamics of the source ( 10's keV) and seed ( $\sim 100$ 's keV) populations of the radiation belts were simulated with RAMSCB and compared with complementary data sets from the Van Allen Probes in the morning sector and NOAA-15 satellite in the predawn and afternoon MLT sectors. It was found that the simulated electron flux at lower $(E<100$ $\mathrm{keV}$ ) energies was in good agreement with observations of both trapped and precipitating electron fluxes. The flux increased during both SYM-H dips and decreased during the intermediate recovery phase due to time-dependent magnetospheric convection, showing the initial formation of an asymmetric ring current that evolved into a more symmetric one with storm development. The injection of high-energy electrons, however, was underestimated by convective transport throughout the storm. Local acceleration of freshly injected electrons by chorus waves intensified the electron fluxes at $E \geq 50 \mathrm{keV}$ considerably during the first SYM-H dip and the simulations overestimated the trapped fluxes observed with Van Allen Probes by more than an order of magnitude (Figure 5. bottom). The precipitating fluxes simulated with RAM-SCB were weaker and their temporal and spatial evolution agreed well with NOAA/POES data (see Figures 3,10 and 11 of [50]).

The significant electron acceleration by plasma waves seen in RAM-SCB simulations at energies as low as $\sim 50 \mathrm{keV}$ was not expected initially since the energy diffusion coefficients are much smaller than the pitch angle diffusion coefficients in this energy range (Figure 5 top). The presence of a large energy gradient that developed in the electron phase space distribution at the injection boundary after particles were injected from the nightside, enhanced this energization effect [50]. These results indicate that either: a) including additional loss mechanisms (such as enhanced losses through the dayside magnetopause or Coulomb collisions), or b) an improved representation of plasma wave scattering and possibly taking into account nonlinear effects [53] are needed to better reproduce the relativistic electron populations of the near-Earth space environ350 ment.

In order to develop a more self-consistent treatment of wave-particle interac- 


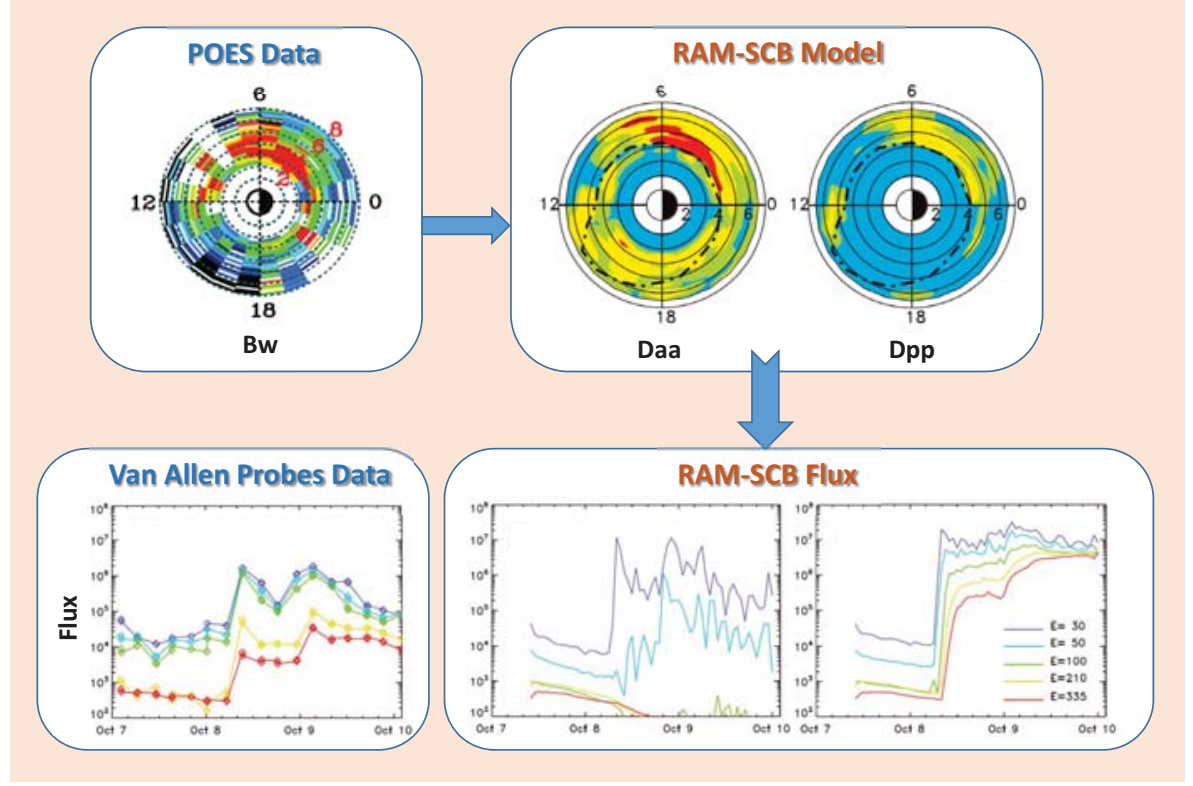

Figure 5: (top) Schematic representation of RAM-SCB model driven by event-specific plasma wave models from POES and Van Allen Probes data. (bottom) Comparison of Van Allen Probes flux data (left) with RAM-SCB simulations (middle) without and (right) with local acceleration by plasma waves. $\mathrm{Bw}$ - wave amplitude, Daa - quasi-linear pitch angle diffusion coefficient, Dpp - momentum diffusion coefficient. 
tions, we investigated the coupling of the large-scale kinetic RAM-SCB model with a very high resolution Particle-In-Cell (PIC) code. PIC simulations allow the study of the growth of kinetic plasma instabilities and the saturation mechanisms via nonlinear effects including wave-particle interactions, which are essential for inner magnetosphere dynamics. In an initial study, we identified regions of enhanced plasma instability based on calculations of linear growth rate of whistler-mode chorus with the RAM-SCB model during a storm that occurred on 23-24 October 2002 [47]. Using unstable electron distributions from RAM-SCB simulations as input to the iPIC3D code [9], we found that whistlermode waves were excited and grew exponentially, propagating mainly along the background magnetic field. The high anisotropy of hot (10's keV) electrons distinctly dropped when the waves were fully developed, and these whistler-mode waves were subsequently damped by the cooler (few $\mathrm{keV}$ ) electron population [54]. Our simulations verified that the waves generated from RAM-SCB's most anisotropic electron distributions are broadly consistent with the largest waveamplitudes that are observed during storms. Moreover, the locations and times of these predicted, most unstable distributions are very consistent with satellite observations. Future extensions of this work will consider incorporating the feedback from these PIC simulations into the RAM-SCB model.

\subsection{Data assimilation}

As part of SHIELDS, we have built a data assimilation scheme [11] to improve the specification of the SCE. Input parameters in physics-based models (i.e., initial and boundary conditions) suffer from errors which eventually result 375 in inaccuracies in the model results. The assimilation procedure can correct for these errors and/or missing physics processes within the model. Data assimilation has proven to be a robust method for reconstructing the radiation belt environment [55, 56], but there have only been a few attempts to apply the approach to the ring current environment [57, 58]. In SHIELDS, we use data assimilation to combine the physics-based transport model RAM-SCB with in situ observational data obtained from near-Earth orbiting satellites. Our ap- 

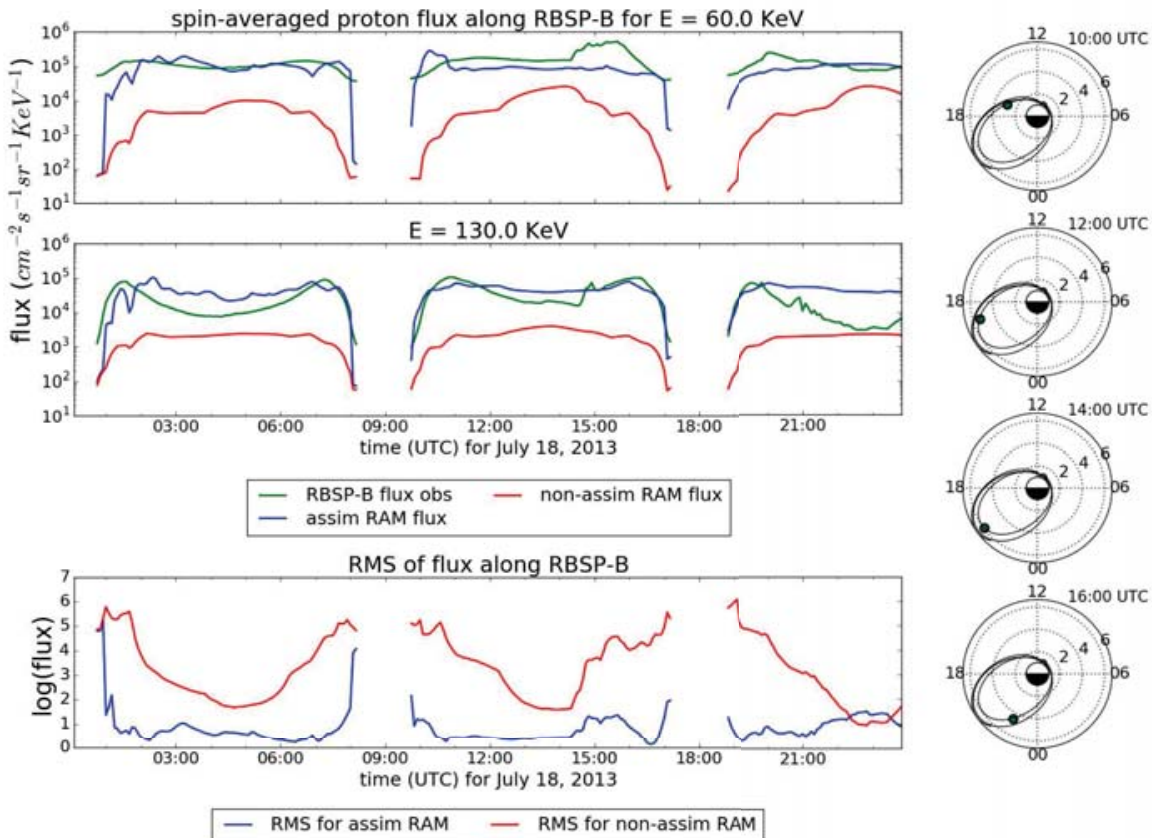

12 14:00 UTC

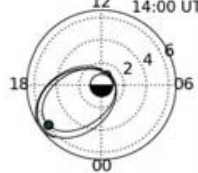

12 16:00 UTC

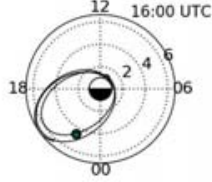

Figure 6: Spin-averaged flux spectrogram for observations from RBSP-B (green line), assimilated RAM-SCB results using RBSP-A observations (blue line), and unassimilated RAM-SCB (red line) during 18 July 2013 for an energy spectrum of $60 \mathrm{KeV}$ (top plot) and $130 \mathrm{KeV}$ (middle plot). Root-mean-square error of the flux along the RBSP-B satellite (bottom plot). The right plots show the trajectory of the Van Allen Probe B (solid black line) and its current position (green dot) for a number of times.

proach is a variant of the ensemble Kalman filter (EnKF) [59]. We modify the basic method by performing the assimilation on an orthonormal projection of the RAM-SCB state space that captures the dominant modes of variation within the model. This projection, which is similar to principal component analysis or empirical orthogonal functions, helps prevent the data from pushing the model state into unstable regions. To ensure robustness, we also apply an adaptive inflation technique [60] as well as a localization approach [61].

Figure 6 shows the results of our approach for the enhancement of the ring current following the isolated substorm event on 18 July 2013. Here, we are correcting the state of the ring current using proton flux data from Van Allen 
Probe A and comparing the assimilated results with data from Van Allen Probe B. The top two panels show two energy bins of the Van Allen Probe B data in green, the unassimilated RAM-SCB state in red, and the estimated state from our approach in blue. It is clear that the assimilation provides a significant improvement by moving the model state much closer to the data. The bottom panel shows the normalized root mean squared (RMS) error or log-flux for the unassimilated and assimilated RAM-SCB estimates; this error provides a percentage of how much the model deviates from the observations. Over the bulk of this data, our approach reduces the error in flux by at least an order of magnitude and often several orders of magnitude, depending on the time and location. These results indicate that the data assimilation scheme is a promising approach for capturing injection phenomena and improving estimates of the SCE.

\subsection{Surface charging}

SHIELDS can also calculate the surface charging of a spacecraft with any geometry by means of an electrostatic particle-in-cell code known as Curvilinear PIC (CPIC) 62, 63]. Problems involving the interaction of complex material objects with plasmas are challenging computationally because of their multiscale nature: the characteristic spatial (and possibly temporal) scales of the object must also be resolved in the simulation, thus requiring additional sophistication compared to standard PIC methods. For instance, at geosynchronous orbit the characteristic scales of the plasma like the electron Debye length or the electron gyroradius are hundreds of meters while the spacecraft characteristic size is on the order of meters, with features that could be even smaller. For these reasons, most tools developed for spacecraft charging calculations [64, 65, 62, 63] use non-uniform, adaptive meshes which conform to the surface of the spacecraft.

Unlike other spacecraft-charging tools, CPIC uses multi-block structured curvilinear meshes to maintain the flexibility necessary to describe complex geometries while guaranteeing the best computational performance. In addition, CPIC uses advanced algorithms and runs efficiently on high-performance 
computing platforms. These algorithms include a mimetic discretization of Poisson's equation suitable for full discontinuous metric tensors found in multi-block meshes, and a scalable solver for the electrostatic field based on the multigrid technique that is critical for performance. Furthermore, CPIC features an asynchronous, hybrid particle mover to minimize the cost of particle tracking which can be a significant burden from PIC methods on unstructured meshes. Details on the CPIC algorithm and the challenges associated with the development of a PIC code on multi-block structured meshes can be found in [63]. CPIC has been applied to several plasma-material interaction problems, including studies of the interaction between a magnetospheric spacecraft and a high-power electron beam 66] and the interaction of dust particles with magnetic fusion energy plasmas [67].

Figure 7 shows an example of applying CPIC to study the surface charging of a geometry representative of the Van Allen Probes. Here, the electrostatic potential is shown for a computational domain that conforms exactly to the surface of the spacecraft. In this illustrative example, the background parameters were taken from SHIELDS simulations for the March 17, 2013 storm. Perfectly conducting boundary conditions at the spacecraft surface were applied for the electrostatic field. This type of simulation provides the electric field and particle flux distribution around the spacecraft and enables us to investigate the connections between the occurrence of anomalies and SCE dynamics. Note that when applying any of the spacecraft-charging codes to anomaly resolution, the biggest uncertainties are in the spacecraft material properties (due to exposure to the harsh space environment) and in the properties of the environment. The SHIELDS framework provides a more accurate description of the space environment, and one can assess whether this exceeds the specifications of the pre-launch simulations when attempting to find the most-probable cause of an anomaly. The specification of the material properties is a much harder problem. An approach that is currently being pursued within SHIELDS is to estimate the material properties by using non-linear optimization to combine Van Allen Probes spacecraft-potential data with spacecraft-charging calcula- 

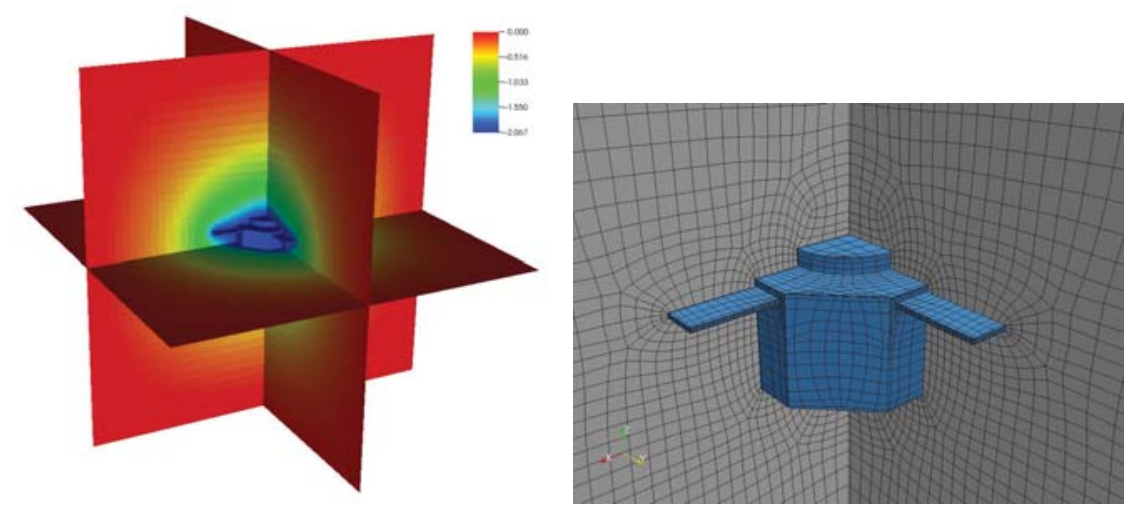

Figure 7: These images show SHIELDS' CPIC calculations of spacecraft charging. On the left, the electrostatic potential (normalized to $5 \mathrm{eV}$ ) around the Van Allen Probes spacecraft arising from the plasma environment obtained by SHIELDS on March 17th 2013. On the right, part of the curvilinear multi-block mesh conforming to the surface of the Van Allen Probes spacecraft.

tions. This approach can yield valuable information on how materials change in space, but one can also envision using data sampled over a selected time period to characterize material properties and then predict spacecraft charging over a later time period.

\subsection{Configuration and testing}

Because of the computational complexity of the multiscale, multiphysics problem that SHIELDS solves, the SHIELDS framework is designed to run at large scale on the world's most powerful supercomputers. For such advanced applications, the SHIELDS software can be built from source following the instructions detailed in the user manual (visit SHIELDS public website http://www.lanl.gov/projects/shields/ for details). However, a critical part of the SHIELDS design philosophy is to allow both users and developers to get working with SHIELDS on any system without having to deal with complicated software dependencies. As such, the SHIELDS framework has been packaged in a Docker 68] image, allowing installation and deployment with a single command on any laptop, desktop, or high-performance computing (HPC) system 
with Docker installed. While the SHIELDS Docker image is not intended for full scale productional simulations on a traditional HPC system, it can be used for rapid software prototyping and for input parameter studies. In addition, to streamline the setup process and to reduce input errors, we have developed an Easy-to-use Graphical Interface for SHIELDS (AEGIS). This tool allows users to select their components of interest, set relevant parameters, and generate various input files and execution scripts needed to achieve a desired modeling result. A layout of SHIELDS installation, configuration, and execution is shown in Figure 8. The SHIELDS application is kept automatically up to date with the latest SHIELDS developments. As changes are made to the SHIELDS code-base, they are automatically built and tested using Travis Continuous Integration [69]. If all tests pass, a new Docker image is built reflecting the code changes and pushed to Dockerhub. SHIELDS framework releases are automatically tagged with the corresponding change to the source code. Examples of how to use different elements of the SHIELDS framework are provided in the user manual.

We are developing a near real-time version of the SHIELDS framework that allows for the simulation of only the inner magnetosphere plasma environment using RAM-SCB. An option exists to output spacecraft-charging environment spectra and plot them along given satellite trajectory. This mode is best suited for long term simulations with operational implications (e.g. monitoring Dst, satellite-specific charging environments, etc.). In this initial version, SCB is deactivated and a simple dipole magnetic field is used together with a VollandStern electric field. The external boundary conditions are provided by newlydeveloped models [70, 71], capable of predicting electron and ion fluxes at GEO with about an hour of lead time using upstream solar wind data, and several hours of lead time using a prediction of $K p$ to drive the model. Figure 9 shows an example output from a simulation run during February 2017 using $K p$ as a model driver and providing a 3 hour SCE prediction along Van Allen Probe's orbit. 


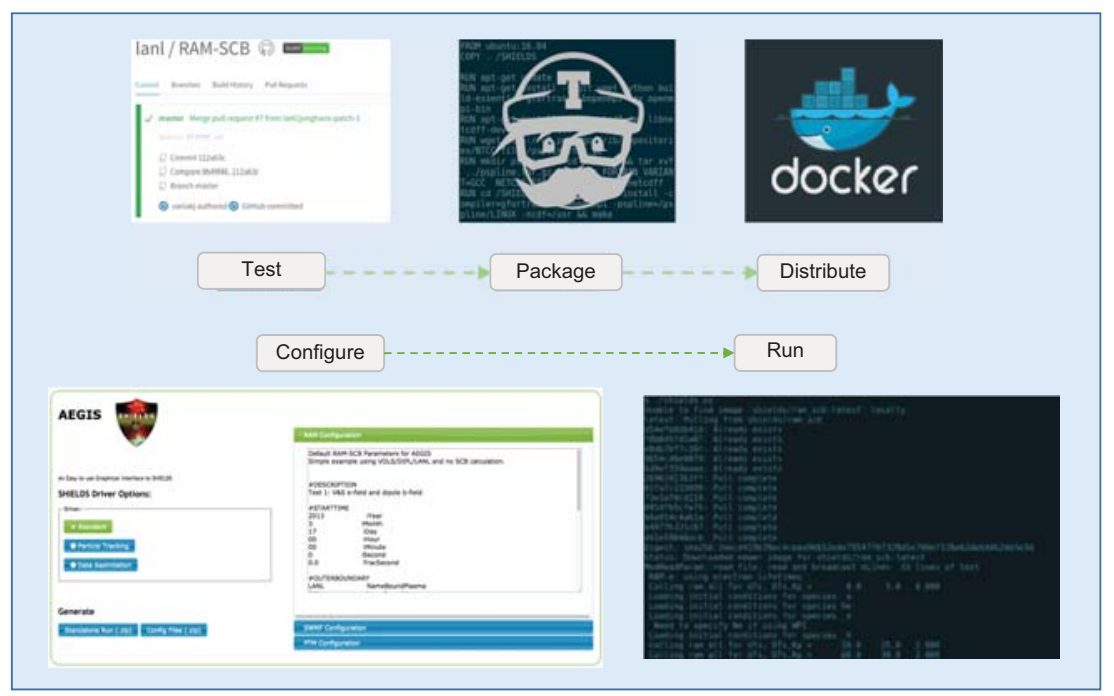

Figure 8: This figure demonstrates the automated workflow behind the compilation, testing and deployment of SHIELDS. By packaging of SHIELDS with Docker and AEGIS we enable rapid deployment and execution of SHIELDS on local machines, traditional clusters and cloud infrastructure. 


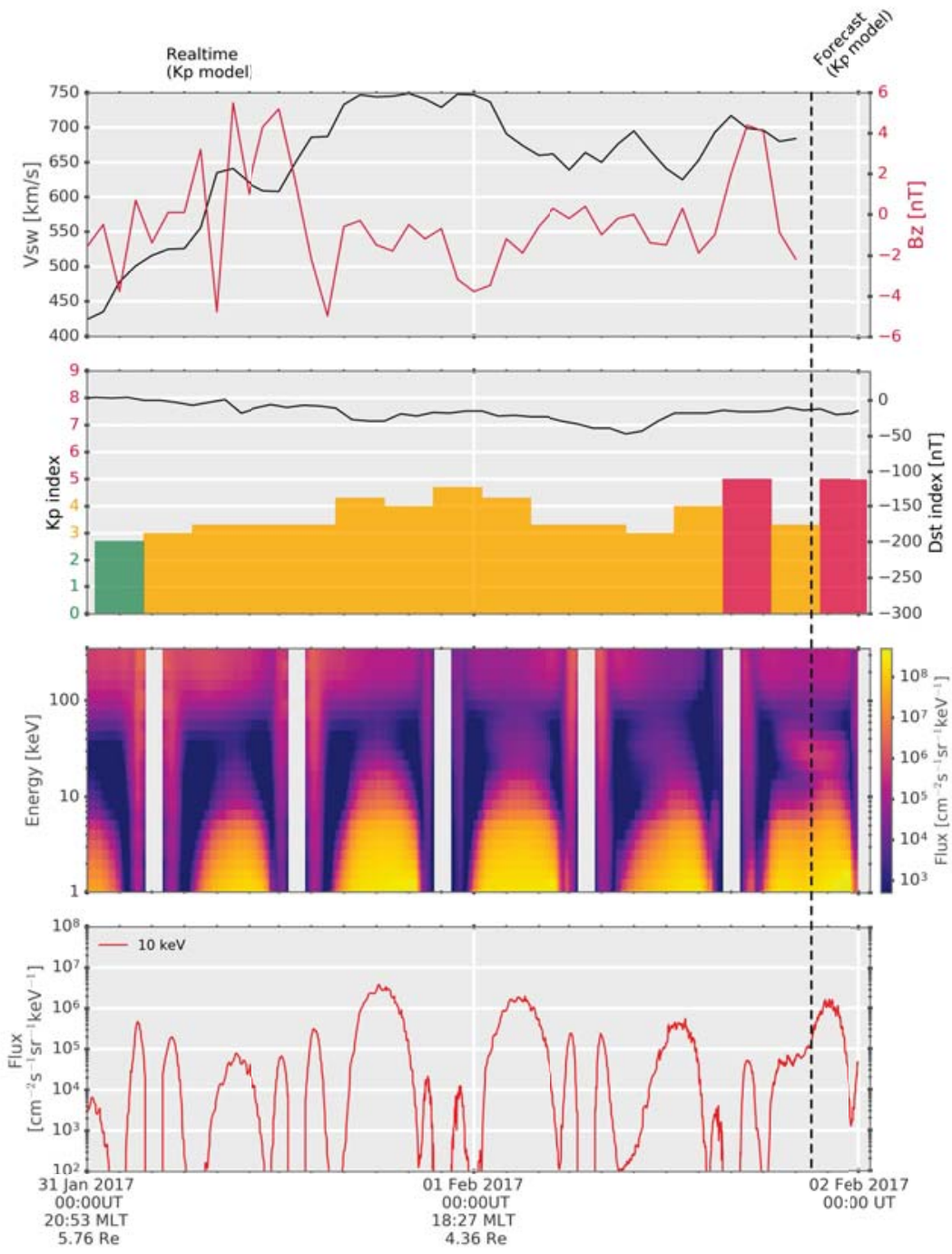

Figure 9: Output of the SHIELDS near real-time model showing a 3 hour SCE prediction along Van Allen Probe-A satellite trajectory during 01 Feb 2017. From top to bottom the panels show solar wind velocity and IMF $B_{z}$, the $K p$ and $D s t$ indices, simulated electron flux spectrogram, and omnidirectional electron flux at $10 \mathrm{keV}$. 


\section{Summary and Conclusions}

500

The emerging research field of space weather is rapidly gaining importance and public recognition due to its technological and societal impact. Government agencies around the world have issued comprehensive reports and studies related to the space environment and space weather [1, 5]. These urge to create a response and recovery plan in the event of extreme space weather. Important components of this plan include developing predictive models and transitioning space weather models from research to operations.

From the variety of space weather phenomena that can have impacts on operating space systems, one of the major concerns is spacecraft surface charging. The SHIELDS framework developed at LANL addresses this spacecraft hazard.

10 It employs multiscale modeling and assimilates in situ satellite data to achieve a realistic description of the inner magnetosphere, enabling a better prediction of the surface charging environment (SCE). Magnetospheric disturbance events like storms and substorms are a primary cause of SCE enhancement. During such space weather events, low-energy (10's of keV) hot particles are injected from the Earth's magnetotail into the inner magnetosphere. We have designed SHIELDS to simulate the dynamics of such hot particles using advanced scientific and computational algorithms. Major improvements of the SHIELDS framework are:

1) Successful coupling of a global MHD (BATS-R-US) code with a particlein-cell (iPIC3D) code; this coupling represents the most efficient approach to resolving magnetic reconnection physics and substorm dynamics in a global system.

2) Achieving the first data assimilation for the inner magnetosphere model RAM-SCB, demonstrating an order of magnitude improvement in the accuracy in the simulation of the spacecraft surface charging environment.

3) Developing a more self-consistent treatment of global transport and WPI that helps unraveling the physics of cross-energy coupling in the Earth's radiation belts. It showed that the acceleration of freshly injected electrons by 
plasma waves at injection boundaries may be significant at energies as low as ${ }_{530} \sim 50 \mathrm{keV}$, thus affecting the SCE.

4) Including a post-processing tool designed to calculate the surface charging for specific spacecraft geometry using the CPIC code and evaluate anomalies' relation to SCE dynamics. Such diagnostics are critically important when performing forensic analyses of space-system failures.

Acknowledgments This research was conducted as part of the Space Hazards Induced near Earth by Large, Dynamic Storms (SHIELDS) project, funded by the U.S. Department of Energy through the LANL/LDRD-DR Program under contract DE-AC52-06NA25396. We thank J. Borovsky, M. Thomsen, C. Lemon, and S. Young for helpful discussions and R. Skoug for providing solar wind data from the ACE satellite to cover gaps in the publicly available Level 2 datasets. The geomagnetic indices and solar wind observation data are obtained from NASA GSFC OMNIWeb, while NOAA F10.7 flux is obtained from http://lasp.colorado.edu/lisird/tss/noaa_radio_flux.html.

\section{References}

[1] D. N. Baker, L. J. Lanzerotti, Resource Letter SW1: Space Weather, Am. J. Phys. 84 (2016) 166. doi:10.1119/1.4938403,

[2] R. B. Horne, D. Pitchford, Space Weather Concerns for All-Electric Propulsion Satellites, Space Weather 13 (2015) 1. doi:10.1002/2015SW001198.

[3] D. N. Baker, et al., Severe space weather events - understanding societal and economic impacts workshop report, National Research Council Technical Report (2008) ISBN 0-309-12770-X.

[4] R. B. Horne, S. A. Glauert, N. P. Meredith, D. Boscher, V. Maget, D. Heynderickx, D. Pitchford, Space weather impacts on satellites and forecasting the Earth's electron radiation belts with SPACECAST, Space Weather 11 (2013) 1. doi:10.1002/swe.20023. 
[5] P. S. Cannon, Extreme Space WeatherA Report Published by the UK Royal Academy of Engineering, Space Weather 11 (2013) 138139. doi:10.1002/swe.20032.

[6] P. B. Selding, Orbital's Revenue Hits Record High but Earnings Fall, Space News (2010) Jul 22.

[7] J. F. Fennell, H. C. Koons, J. L. Roeder, J. B. Blake, Spacecraft charging: Observations and relation-ship to satellite anomalies, ESA Special Publications SP-476 (2001) 279.

[8] G. Tóth, et al., Space Weather Modeling Framework: A new tool for the space science community, J. Geophys. Res. 110 (A12) (2005) n/a. doi:10.1029/2005JA011126.

[9] S. Markidis, G. Lapenta, Rizwan-uddin, Multi-scale simulations of plasma with iPIC3D, Mathematics and Computers in Simulation 80 (2010) 15091519 .

[10] V. K. Jordanova, S. Zaharia, D. T. Welling, Comparative study of ring current development using empirical, dipolar, and self-consistent magnetic field simulations, J. Geophys. Res. 115 (2010) A00J11. doi:10.1029/2010JA015671.

[11] H. C. Godinez, Y. Yu, E. Lawrence, M. G. Henderson, B. Larsen, V. K. Jordanova, Ring current pressure estimation with RAM-SCB using data assimilation and Van Allen Probe flux data, Geophys. Res. Lett. 43 (2016) n/a. doi:10.1002/2016GL071646.

[12] K. G. Powell, P. L. Roe, T. J. Linde, T. I. Gombosi, D. L. D. Zeeuw, A solution-adaptive upwind scheme for ideal magnetohydrodynamics, J. Comp. Phys. 154 (1999) 284.

[13] G. Tóth, B. van der Holst, I. V. Sokolov, D. L. D. Zeeuw, T. I. Gombosi, et al., Adaptive Numerical Algorithms in Space Weather Modeling, J. Comp. Phys. 231 (2012) 870. doi:10.1016/j.jcp.2011.02.006 
[14] A. Ridley, T. Gombosi, D. D. Zeeuw, Ionospheric control of the magnetosphere: conductance, Ann. Geophys. 22 (2004) 567-584.

[15] L. Daldorff, G. Tóth, T. I. Gombosi, G. Lapenta, J. Amaya, S. Markidis, J. U. Brackbill, Two-way coupling of a global Hall magnetohydrodynamics model with a local implicit Particle-in-Cell model, J. Comput. Phys. 268 (2014) 236. doi:10.1016/j.jcp.2014.03.009

[16] G. Tóth, X. Jia, S. Markidis, B. Peng, Y. Chen, L. Daldorff, V. Tenishev, D. Borovikov, J. Haiducek, T. Gombosi, A. Glocer, J. Dorelli, Extended Magnetohydrodynamics with Embedded Particle-in-Cell Simulation of Ganymede's Magnetosphere, J. Geophys. Res. 121 (2016) n/a. doi:10.1002/2015JA021997.

[17] Y. Chen, G. Tóth, P. Cassak, X. Jia, T. I. Gombosi, J. A. Slavin, S. Markidis, I. B. Peng, V. K. Jordanova, M. G. Henderson, Global threedimensional simulation of Earth's dayside reconnection using a two-way coupled magnetohydrodynamics with embedded particle-in-cell model: initial results, J. Geophys. Res. 122 (2017) -. doi:10.1002/2017JA024186.

[18] G. Tóth, Y. Chen, T. I. Gombosi, P. Cassak, S. Markidis, I. B. Peng, Scaling the ion inertial length and its implications for modeling reconnection in global simulations, J. Geophys. Res. 122 (2017) n/a. doi:10.1002/2017JA024189.

[19] N. Yokoyama, Y. Kamide, Statistical nature of geomagnetic storms, J. Geophys. Res. 102 (A7) (1997) 14215. doi:10.1029/97JA00903.

[20] W. Baumjohann, M. Hesse, S. Kokubun, T. Mukai, T. Nagai, A. A. Petrukovich, Substorm dipolarization and recovery, J. Geophys. Res. 104 (A11) (1999) 24995. doi:10.1029/1999JA900282.

[21] J. E. Borovsky, R. J. Nemzek, R. D. Belian, The occurrence rate of magnetospheric-substorm onsets: Random and periodic substorms, J. Geophys. Res. 98 (A3) (1993) 3807. doi:10.1029/92JA02556. 
[22] D. L. De Zeeuw, S. Sazykin, R. A. Wolf, T. I. Gombosi, A. J. Ridley, G. Tóth, Coupling of a global MHD code and an inner magnetospheric model: Initial results, J. Geophys. Res. 109 (2004) A12219. doi:10.1029/2003JA010366.

[23] Y. Yu, V. Jordanova, D. Welling, B. Larsen, S. G. Claudepierre, C. Kletzing, The role of ring current particle injections: Global simulations and Van Allen Probes observations during 17 March 2013 storm, Geophys. Res. Lett. 41 (2014) 1126. doi:10.1002/2014GL059322.

${ }_{620}[24]$ T. I. Pulkkinen, D. N. Baker, M. Wiltberger, C. Goodrich, R. E. Lopez, J. G. Lyon, Pseudobreakup and substorm onset: Observations and MHD simulations compared, J. Geophys. Res. 103 (A7) (1998) 14847-14854. doi:10.1029/97JA03244.

[25] D. T. Welling, A. J. Ridley, Validation of SWMF magnetic field and plasma, Space Weather 8 (2010) n/a. doi:10.1029/2009SW000494

[26] A. Pulkkinen, L. Rastätter, M. Kuznetsova, H. Singer, C. Balch, D. Weimer, G. Toth, A. Ridley, T. Gombosi, M. Wiltberger, J. Raeder, R. Weigel, Community-wide validation of geospace model ground magnetic field perturbation predictions to support model transition to operations, Space Weather 11 (6) (2013) 369-385. doi:10.1002/swe.20056.

[27] D. J. McComas, S. J. Bame, P. Barker, W. C. Feldman, J. L. Phillips, P. Riley, J. W. Griffee, The Advanced Composition Explorer Mission, Springer Netherlands, Dordrecht, 1998, Ch. Solar Wind Electron Proton Alpha Monitor (SWEPAM) for the Advanced Composition Explorer, pp. 563-612. doi:10.1007/978-94-011-4762-0\_20

[28] X. Chu, R. L. McPherron, T.-S. Hsu, V. Angelopoulos, Solar cycle dependence of substorm occurrence and duration: Implications for onset, Journal of Geophysical Research: Space Physics 120 (4) (2015) 2808-2818. doi:10.1002/2015JA021104. 
[29] H. U. Frey, S. B. Mende, V. Angelopoulos, E. F. Donovan, Substorm onset observations by image-fuv, Journal of Geophysical Research: Space Physics 109 (A10) (2004) n/a-n/a, a10304. doi:10.1029/2004JA010607.

[30] R. D. Belian, G. R. Gisler, T. Cayton, R. Christensen, High-z energetic particles at geosynchronous orbit during the great solar proton event series of october 1989, Journal of Geophysical Research: Space Physics 97 (A11) (1992) 16897-16906. doi:10.1029/92JA01139.

[31] H. Singer, L. Matheson, R. Grubb, A. Newman, D. Bouwer, Monitoring space weather with the GOES magnetometers, Proc. SPIE 2812 (1996) 299-308. doi:10.1117/12.254077.

[32] J. N. Barfield, C. S. Lin, R. L. McPherron, Observations of magnetic field perturbations at GOES 2 and GOES 3 during the March 22, 1979, Substorms: CDAW 6 analysis, J. Geophys. Res. 90 (A2) (1985) 1289. doi:10.1029/JA090iA02p01289.

[33] S. Morley, J. Koller, D. Welling, B. Larsen, J. Niehof, SpacePy: PythonBased Tools for the Space Science Community, Astrophysics Source Code Library (Jan. 2014). arXiv:1401.002.

[34] D. S. Wilks, Statistical methods in the atmospheric sciences, 2nd Edition, Academic Press, 2006.

[35] D. B. Stephenson, Use of the Odds Ratio for Diagnosing Forecast Skill, Wea. Forecasting 15 (2) (2000) 221-232. doi:10.1175/1520-0434(2000)

[36] D. T. Welling, M. W. Liemohn, The ionospheric source of magnetospheric plasma is not a black box input for global models, J. Geophys. Res. 121 (6) (2016) 5559. doi:10.1002/2016JA022646.

[37] E. A. Kronberg, et al., Circulation of Heavy Ions and Their Dynamical Effects in the Magnetosphere: Recent Observations and Models, Space Science Reviews 184 (1) (2014) 173-235. doi:10.1007/s11214-014-0104-0. 
[38] J. Birn, M. F. Thomsen, M. Hesse, Electron acceleration in the dynamic magnetotail: Test particle orbits in three-dimensional magnetohydrodynamic simulation fields, Phys. Plas. 11 (2004) -. doi:10.1063/1.1704641.

[44] R. B. Horne, R. M. Thorne, Potential waves for relativistic electron scattering and stochastic acceleration during magnetic storms, Geophys. Res. Lett. 25 (1998) 3011.

[45] N. P. Meredith, R. B. Horne, A. Sicard-Piet, D. Boscher, K. H. Yearby, 690 W. Li, R. M. Thorne, Global model of lower band and upper band chorus from multiple satellite observations, J. Geophys. Res. 117 (2012) A10225. doi:10.1029/2012JA017978

[46] W. Li, Q. Ma, R. M. Thorne, J. Bortnik, C. A. Kletzing, W. S. Kurth, G. B. Hospodarsky, Y. Nishimura, Statistical properties of plasmas- 
pheric hiss derived from Van Allen Probes data and their effects on radiation belt electron dynamics, J. Geophys. Res. 120 (2015) 33933405. doi:10.1002/2015JA021048,

[47] V. K. Jordanova, D. T. Welling, S. Zaharia, L. Chen, R. M. Thorne, Modeling ring current ion and electron dynamics and plasma instabilities during a high-speed stream driven storm, J. Geophys. Res. 117 (2012) A00L08. doi:10.1029/2011JA017433.

[48] S. Zaharia, V. K. Jordanova, D. T. Welling, G. Tóth, Self-consistent inner magnetosphere simulation driven by a global MHD model, J. Geophys. Res. 115 (2010) A12228. doi:10.1029/2010JA015915.

[49] Y. Yu, V. K. Jordanova, A. J. Ridley, J. M. Albert, R. B. Horne, C. A. Jeffery, A new ionospheric electron precipitation module coupled with RAMSCB within the geospace general circulation model, J. Geophys. Res. 121 (2016) 8554. doi:10.1002/2016JA022585.

[50] V. K. Jordanova, W. Tu, Y. Chen, S. K. Morley, A.-D. Panaitescu, G. D. Reeves, C. A. Kletzing, RAM-SCB simulations of electron transport and plasma wave scattering during the October 2012 "double-dip" storm, J. Geophys. Res. 121 (2016) 8712. doi:10.1002/2016JA022470

[51] N. P. Meredith, R. B. Horne, R. M. Thorne, D. Summers, R. R. Anderson, Substorm dependence of plasmaspheric hiss, J. Geophys. Res. 109 (2004) A06209. doi:10.1029/2004JA010387.

[52] W. Tu, G. S. Cunningham, Y. Chen, M. G. Henderson, E. Camporeale, G. D. Reeves, Modeling radiation belt electron dynamics during GEM challenge intervals with the DREAM3D diffusion model, J. Geophys. Res. 118 (2013) n/a. doi:10.1002/jgra.50560

[53] J. M. Albert, X. Tao, J. Bortnik, Dynamics of the Earth's Radiation Belts and Inner Magnetosphere, Vol. 199, Geophys. Monogr. Ser., 
2012, Ch. Aspects of Nonlinear Wave-Particle Interactions, p. 255. doi:10.1029/2012BK001324.

[54] Y. Yu, G. L. Delzanno, V. K. Jordanova, I. B. Peng, S. Markidis, PIC simulations of wave-particle interactions initialized by a physics-based electron distribution from the RAM-SCB model, J. Atm. Sol.-Terr. Phys. in press.

[55] S. Bourdarie, R. H. W. Friedel, J. Fennell, S. Kanekal, T. E. Cayton, Radiation belt representation of the energetic electron environment: Model and data synthesis using the Salammb radiation belt transport code and Los Alamos geosynchronous and GPS energetic particle data, Space Weather 3 (2005) S04S01. doi:10.1029/2004SW000065.

[56] H. C. Godinez, J. Koller, Localized adaptive inflation in ensemble data assimilation for a radiation belt model, Space Weather 10 (2012) S08001. doi:10.1029/2012SW000767.

[57] T. W. Garner, R. A. Wolf, R. W. Spiro, First attempt at assimilating data to constrain a magnetospheric model, J. Geophys. Res. 104 (1999) $25,14525,152$.

[58] S. Nakano, G. Ueno, Y. Ebihara, M.-C. Fok, S. Ohtani, P. C. Brandt, D. G. Mitchell, K. Keika, T. Higuchi, A method for estimating the ring current structure and the electric potential distribution using energetic neutral atom data assimilation, J. Geophys. Res. 113 (2008) A05208. doi:10.1029/2006JA011853.

[59] G. Evensen, The Ensemble Kalman Filter: Theoretical formulation and practical implementation, Ocean Dyn. 53 (2003) 343367. doi:10.1007/s10236-003-0036-9.

[60] H. Li, E. Kalnay, T. Miyoshi, Simultaneous estimation of covariance inflation and observation errors within an ensemble Kalman filter, Q. J. R. Meteorol. Soc. 135 (2009) 523533. 
[61] T. Hamill, J. Whitaker, C. Snyder, Distance-dependent filtering of background error covariance estimates in an ensemble Kalman filter, Mon. Weather Rev. 129 (2001) 27762790.

[62] G. L. Delzanno, E. Camporeale, J. D. Moulton, J. E. Borovsky, E. A. MacDonald, M. F. Thomsen, CPIC: a curvilinear particle-in-cell code for plasma-material interaction studies, IEEE Trans. Plas. Sci. 41 (2013) 3577.

[63] C. S. Meierbachtol, D. Svyatsky, G. L. Delzanno, L. J. Vernon, J. D. Moulton, An Electrostatic Particle-In-Cell Code on Multi-block Structured Meshes, J. Comput. Phys. 350 (2017) 796-823.

[64] M. J. Mandell, V. A. Davis, D. L. Cooke, A. T. Wheelock, C. J. Roth, Nascap-2k spacecraft charging code overview, IEEE Transactions on Plasma Science 34 (2006) 2084.

[65] J. F. Roussel, F. Rogier, G. Dufour, J. C. Mateo-Velez, J. Forest, A. Hilgers, D. Rodgers, L. Girard, D. Payan, Spis open-source code: Methods, capabilities, achievements, and prospects, IEEE Transactions on Plasma Science 36 (2008) 2360.

[66] G. L. Delzanno, J. E. Borovsky, M. F. Thomsen, J. D. Moulton, E. A. MacDonald, Future beam experiments in the magnetosphere with plasma contactors: How do we get the charge off the spacecraft?, J. Geophys. Res. 120 (2015) 3647. doi:10.1002/2014JA020608.

[67] G. L. Delzanno, X.-Z. Tang, Charging and heat collection by a positively charged dust grain in a plasma, Phys. Rev. Lett. 113 (2014) 035002.

[68] D. Merkel, Docker: Lightweight linux containers for consistent development and deployment, Linux J. 2014 (239). URL http://dl.acm.org/citation.cfm?id=2600239.2600241

[69] G. Booch, Object Oriented Design with Applications, Benjamin-Cummings Publishing Co., Inc., Redwood City, CA, USA, 1991. 
[70] M. H. Denton, M. F. Thomsen, V. K. Jordanova, M. G. Henderson, J. E. Borovsky, J. S. Denton, D. Pitchford, D. P. Hartley, An empirical model of electron and ion fluxes derived from observations at geosynchronous orbit, Space Weather 13 (2015) n/a. doi:10.1002/2015SW001168.

[71] M. H. Denton, M. G. Henderson, V. K. Jordanova, M. F. Thomsen, J. E. Borovsky, J. Woodroffe, D. P. Hartley, D. Pitchford, An improved empirical model of electron and ion fluxes at geosynchronous orbit based on upstream solar wind conditions, Space Weather 14 (2016) n/a. doi:10.1002/2016SW001409 